\title{
On the Break-down of Completeness of Wave Operators in Potential Scattering
}

\author{
D. R. Yafaev*
}

St. Banach International Mathematical Center, Warsaw, Poland, and Leningrad Department of Mathematical Institute, Leningrad, USSR

\begin{abstract}
We study the Schrödinger operator with a potential that vanishes at infinity but the rate of falloff of the potential depends on the direction. It turns out that for such potentials scattering theory becomes in general multichannel.
\end{abstract}

\section{Introduction}

It is well-known that the continuous spectrum of the self-adjoint Schrödinger operator $H=-\Delta+q$ in the space $\mathscr{H}=L_{2}\left(\mathbb{R}^{m}\right)$ coincides with the half-line $[0, \infty)$ if $q(x) \rightarrow 0$ when $|x| \rightarrow \infty$. Moreover, if the estimate

$$
|q(x)| \leqq C(1+|x|)^{-1-\varepsilon}, \quad \varepsilon>0,
$$

is satisfied, then for the pair $H_{0}=-\Delta, H$ the wave operators (WO) exist and are complete [1]. In constructing the scattering theory the estimate (1.1) is in some sense optimal: if $q(x)=c|x|^{-1}$ the WO do not exist. On the other hand, in the theory of multiparticle scattering [2-4] when the potential does not decrease in some directions the WO may exist but may not be complete. The break-down of completeness is connected with the existence of eigenvalues of operators describing subsystems of smaller numbers of particles; thus the continuous spectrum of the operator $H$ covers the half-line $[x, \infty)$ where $x<0$.

This paper is devoted to the study of the Schrödinger operator with a potential that tends to 0 if $|x| \rightarrow \infty$, but the rate of falloff depends on the direction. It appears that for such potentials scattering theory becomes, in general, multichannel. This means that although the WO $W$ may exist, its range $R(W)$ need not coincide with the whole absolute continuous subspace $\mathscr{H}_{a c}$ of the operator $H$. The break-down of completeness of the WO is thereby connected with the following situation, corresponding to the existence of negative eigenvalues for subsystems for multiparticle Schrödinger operators.

* Permanent address: Leningrad Department of Mathematical Institute, USSR Academy of Science, 27, r. Fontanka, 191011 Leningrad, USSR 
Let $\mathbb{R}^{m}=\mathbb{R}^{m_{1}} \oplus \mathbb{R}^{m_{2}}$ and correspondingly $x=\left(x_{1}, x_{2}\right)$. Let us introduce the operator $h\left(x_{2}\right)=-\Delta_{1}+q\left(x_{1}, x_{2}\right)$ acting in the space $\mathscr{H}_{1}=L_{2}\left(\mathbb{R}^{m_{1}}\right)$ and depending on the parameter $x_{2}$. Assume that the operator $h\left(x_{2}\right)$ has negative eigenvalue $\lambda\left(x_{2}\right)$, tending sufficiently slowly (slower than $\left|x_{2}\right|^{-1}$ ) to zero when $\left|x_{2}\right| \rightarrow \infty$. We denote by $\psi\left(x_{1}, x_{2}\right)$ the corresponding eigenfunction of the operator $h\left(x_{2}\right)$. Then under suitable assumptions there exists not only the WO $W$ but also some "additional" isometric WO $w$, and the subspaces $R(W)$ and $R(w)$ are orthogonal. The operator $w$ is constructed in terms of the function $\psi\left(x_{1}, x_{2}\right)$ and some properties of scattering [5] with the auxiliary potential $\lambda\left(x_{2}\right)$ in the space $\mathscr{H}_{2}=L_{2}\left(\mathbb{R}^{m_{2}}\right)$. In contrast to the Schrödinger operator with multiparticle potential, in our situation the operator $H$ has no negative continuous spectrum [because $q(x) \rightarrow 0$ when $|x| \rightarrow \infty$ ], but its negative discrete spectrum is necessarily infinite.

Using multiparticle terminology, let us consider $H$ to be the energy operator of a system of two particles (described correspondingly by coordinates $x_{1}$ and $x_{2}$ ) with the potential energy depending in a sufficiently arbitrary way on the positions of the particles. In these terms the WO $W$ corresponds to the states for which both particles are asymptotically free ("senior" or main channel of scattering). The "additional" WO $w$ describes the process ("junior" channel of scattering), for which the first particle is asymptotically in the state with wave function $\psi\left(x_{1}, x_{2}\right)$ (depending on the position of the second particle), and the second particle is being scattered on the "effective" potential $\lambda\left(x_{2}\right)$.

Earlier the multidimensional Schrödinger operator with anisotropically decreasing potential was examined in $[6,7]$, where it was established that the WO $W$ exist and are complete if

$$
|q(x)| \leqq C\left(1+\left|x_{1}\right|\right)^{-\alpha_{1}}\left(1+\left|x_{2}\right|\right)^{-\alpha_{2}}
$$

and

$$
\alpha_{1}+2^{-1} \min \left\{\alpha_{2}, m_{2}\right\}>1, \alpha_{2}+2^{-1} \min \left\{\alpha_{1}, m_{1}\right\}>1, \alpha_{j}>0 .
$$

At the same time, using Cook's criterion it is easy to verify that under the assumption (1.2) the WO $W$ exist if $\alpha_{1}+\alpha_{2}>1$. The above described effect of the break-down of completeness of $W$ can take place if $\alpha_{1}+\alpha_{2}>1, \alpha_{j} \geqq 0$, but one of the conditions (1.3) is not fulfilled. Therefore the sufficient conditions for completeness of the WO in the papers $[6,7]$ are in some sense close to necessary.

By its subject and methods the present paper is close to the note [8], where the break-down of completeness of the WO in scattering by time-dependent potentials was discovered.

\section{Preliminaries}

Let the operator $H=-\Delta+q, q=\bar{q}$, be self-adjoint in the space $\mathscr{H}=L_{2}\left(\mathbb{R}^{m}\right)$ on the domain of the unperturbed operator $H_{0}=-\Delta$. The wave operator (WO) $W_{ \pm}$for the pair $H_{0}, H$ is defined by the formula

$$
W_{ \pm}=W_{ \pm}\left(H, H_{0}\right)=\underset{t \rightarrow \pm \infty}{s-\lim _{ \pm}} \exp (i H t) \exp \left(-i H_{0} t\right) .
$$

If the limit (2.1) exists then the WO $W_{ \pm}$is isometric and $H W_{ \pm}=W_{ \pm} H_{0}$. The WO is called complete if $R\left(W_{ \pm}\right)=\mathscr{H}_{a c}$. The breakdown of completeness of the WO leads 
to the non-unitarity of the scattering operator $S=W_{+}^{*} W_{-}$. We assume that $t \rightarrow+\infty$ and omit indices " $\pm ",\|\cdot\|=\|\cdot\|_{\mathscr{H}}, \mathscr{H}_{j}=L_{2}\left(\mathbb{R}^{m_{j}}\right), j=1,2,\|\cdot\|_{j}=\|\cdot\|_{\mathscr{H}_{j}}$. An integral without limits means integration over the whole range of variables of integration, $C$ and $\varepsilon$ are different positive numbers (correspondingly sufficiently large and sufficiently small), whose exact values are of no importance for us. We do not assume that $q(x) \rightarrow 0$ when $|x| \rightarrow \infty$. In particular, our results are applicable to decreasing potentials. This case is most interesting.

We need the following well-known assertion [9]. Let $V$ be the operator of multiplication by the function $(1+|x|)^{-1-\varepsilon}, \varepsilon>0$. Then there exists a number $\delta=\delta(\varepsilon, m)>1$ such that for $f \in \mathscr{D}_{m}, \mathscr{D}_{m}$ some set dense in $L_{2}\left(\mathbb{R}^{m}\right)$, the relation

$$
\left\|V \exp \left(-i H_{0} t\right) f\right\|=O\left(t^{-\delta}\right)
$$

is valid. By virtue of the inequality $\left\|V^{s} g\right\| \leqq\|V g\|^{s}\|g\|^{1-s}, s \in[0,1]$, (2.2) implies that for $f \in \mathscr{D}_{m}$

$$
\left\|(1+|x|)^{-\alpha} \exp \left(-i H_{0} t\right) f\right\|=O\left(t^{-\delta}\right), \quad \alpha \in(0,1]
$$

where $\delta, \delta<\alpha$, can be chosen arbitrarily close to $\alpha$.

Theorem 1. Let the potential $q$ satisfy the estimate (1.2), where $\alpha_{1}+\alpha_{2}>1, \alpha_{j} \geqq 0$. Then the WO $W$ exists.

Proof. It is sufficient [9] to verify that for $f$ from some dense subset of $\mathscr{H}$ and $\delta>1$ the relation (2.2) is valid, where $V$ is the operator of multiplication by the function $\left(1+\left|x_{1}\right|\right)^{-\alpha_{1}}\left(1+\left|x_{2}\right|\right)^{-\alpha_{2}}$. The finite sums of functions $f(x)=f_{1}\left(x_{1}\right) f_{2}\left(x_{2}\right)$, where $f_{j} \in \mathscr{D}_{m_{j}}$, are dense in $\mathscr{H}$ and

$$
\left\|V \exp \left(-i H_{0} t\right) f\right\|=\prod_{j=1}^{2}\left\|\left(1+\left|x_{j}\right|\right)^{-\alpha_{j}} \exp \left(i \Delta_{j} t\right) f_{j}\right\|_{j} .
$$

With the help of (2.3) each of the two factors in the right-hand side can be bounded by $t^{-\delta_{J}}$. One can choose $\delta_{1}+\delta_{2}>1$ because $\delta_{j}$ is arbitrarily close to $\alpha_{j}$ and $\alpha_{1}+\alpha_{2}>1$ by assumption.

We need also some information about generalized wave operators from the article [5]. Let, for some $\alpha>0$, the potential and all its partial derivatives of order $x$, where $|x| \leqq\left[2^{-1} m\right]+\left[\alpha^{-1}\right]+2$, satisfy the estimates

$$
\left|D^{x} q(x)\right| \leqq C(1+|x|)^{-\alpha-|x|} .
$$

Let $\mathbf{l}_{1}=0, \mathbf{l}_{j}(\mathbf{p}, t)=q\left(2 \mathbf{p} t+\nabla_{p} \int_{0}^{t} \mathbf{l}_{j-1}(\mathbf{p}, \tau) d \tau\right), \mathbf{p} \in \mathbb{R}^{m}, k=\left[\alpha^{-1}\right]+1$,

$$
U_{0}^{(k)}(t)=\exp \left[-i H_{0} t-i \int_{0}^{t} \mathbf{I}_{k}(-i \nabla, \tau) d \tau\right] .
$$

Then the strong limit

$$
\tilde{W}=\underset{t \rightarrow \infty}{s-\lim _{i}} \exp (i H t) U_{0}^{(k)}(t)
$$

exists, the operator $\tilde{W}$ is isometric and $H \tilde{W}=\tilde{W} H_{0}$. 
The proof of the existence of the limit (2.5) in [5] is based on the relation

$$
\left\|\left[q(x)-\mathbf{l}_{k}(-i \nabla, t)\right] U_{0}^{(k)}(t) f\right\|=O\left(t^{-1-\varepsilon}\right), \quad f \in \mathscr{D}_{m} .
$$

We note that the set $\mathscr{D}_{m}$ is invariant under the action of the operators $D$. Moreover, if the function $v(x)$ satisfies the inequality (1.1), then for $f \in \mathscr{D}_{m}$

$$
\left\|v U_{0}^{(k)}(t) f\right\|=O\left(t^{-1-\varepsilon}\right) .
$$

If only $v(x) \rightarrow 0$ when $|x| \rightarrow \infty$, then for each $f \in L_{2}\left(\mathbb{R}^{m}\right)$

$$
\lim _{t \rightarrow \infty}\left\|v U_{0}^{(k)}(t) f\right\|=0 \text {. }
$$

The proof of the relations (2.7), (2.8) for arbitrary $k$ is analogous to the well-known case $k=1$. In our applications the role of the space $L_{2}\left(\mathbb{R}^{m}\right)$ is played by $L_{2}\left(\mathbb{R}^{m_{2}}\right)$, $x=x_{2}$, and $q(x)$ is replaced by the function $\lambda\left(x_{2}\right)$.

Let us make more precise the "axiomatic" description of the situation in which the break-down of completeness of the WO takes place. The effective construction of classes of potentials, for which the assumptions introduced here are true, will be given in $\S 6$. Assume that for at least sufficiently large $\left|x_{2}\right|$ the operator $h\left(x_{2}\right)=-\Delta_{1}$ $+q\left(x_{1}, x_{2}\right)$ in the space $\mathscr{H}_{1}=L_{2}\left(\mathbb{R}^{m_{1}}\right)$ has an eigenvalue satisfying with some $\alpha>0$ and $m=m_{2}$ the conditions (2.4). If the operator $h\left(x_{2}\right)$ has several (possibly infinitely many) eigenvalues we choose one of them. We denote by $\psi\left(x_{1}, x_{2}\right)$ the corresponding real-valued normalized, i.e. $\int \psi^{2}\left(x_{1}, x_{2}\right) d x_{1}=1$, eigenfunction of the operator $h\left(x_{2}\right)$. The functions $\lambda\left(x_{2}\right)$ and $\psi\left(x_{1}, x_{2}\right)$ are assumed to be smoothly extended to all $x_{2}$.

Let us construct the function $\mathbf{l}_{k}(\mathbf{p}, t), \mathbf{p} \in \mathbb{R}^{m_{2}}$, corresponding to $\lambda\left(x_{2}\right)$. We shall omit the indice $k, \mathbf{l}=\mathbf{l}_{k}, U_{0}(t)=\exp \left[i t \Delta_{2}-i \int_{0}^{t} \mathbf{l}\left(-i \nabla_{2}, \tau\right) d \tau\right]$. Define the isometric operator of identification $J, J: \mathscr{H}_{2} \rightarrow \mathscr{H}$,

$$
(J f)\left(x_{1}, x_{2}\right)=\psi\left(x_{1}, x_{2}\right) f\left(x_{2}\right) .
$$

If the limit

$$
w=\underset{t \rightarrow \infty}{\operatorname{sim}} \lim (i H t) J U_{0}(t)
$$

exists, then the operator $w$ is isometric, $H w=w\left(-\Delta_{2}\right)$ and $R(w) \subset \mathscr{H}_{a c}$. It will be shown in $\S 4$ that the subspaces $R(W)$ and $R(w)$ are orthogonal and hence $R(W) \neq \mathscr{H}_{a c}$. The spectrum of the restriction of the operator $H$ to the subspace $\mathscr{H}_{a c} \ominus R(W)$ contains the half-line $[0, \infty)$, therefore the multiplicity of its positive spectrum is infinite if $m_{2}>1$ and no less than 2 if $m_{2}=1$.

\section{The Existence of the Additional Wave Operator}

We shall need some assumptions on the behavior of the functions $\psi\left(x_{1}, x_{2}\right)$ when $\left|x_{2}\right| \rightarrow \infty$, corresponding, roughly speaking, to certain "auto-modelity" of their construction. In essence these assumptions mean that

$$
\psi\left(x_{1}, x_{2}\right) \sim\left|x_{2}\right|^{-a m_{1} / 2} \varphi\left(\left|x_{2}\right|^{-a} x_{1}\right), \quad a \in[0,1 / 2), \quad\left|x_{2}\right| \rightarrow \infty .
$$


More precisely, denote $\tilde{\psi}\left(x_{1}, x_{2}\right)=x_{1}^{2} \psi\left(x_{1}, x_{2}\right)$ and assume that for some $a \in[0,1 / 2)$

$$
\left.\begin{array}{rl}
\|\tilde{\psi}\|_{1} & =O\left(\left|x_{2}\right|^{2 a}\right), \\
\left\|\nabla_{2} \tilde{\psi}\right\|_{1} & =O\left(\left|x_{2}\right|^{-1+2 a}\right), \\
\left\|\Delta_{2} \tilde{\psi}\right\|_{1} & =O\left(\left|x_{2}\right|^{-2+2 a}\right), \\
\left\|\Delta_{2} \psi\right\|_{1} & =O\left(\left|x_{2}\right|^{-1-\varepsilon}\right), \\
\left\|a\left[2\left\langle\nabla_{1} \psi, x_{1}\right\rangle_{1}+m_{1} \psi\right] x_{2}\left|x_{2}\right|^{-2}+2 \nabla_{2} \psi\right\|_{1} & =O\left(\left|x_{2}\right|^{-1-\varepsilon}\right)
\end{array}\right\}
$$

Here and in the following $\langle\cdot, \cdot\rangle_{j}$ denotes the scalar product in the Euclidean space $\mathbb{R}^{m_{j}}, j=1,2$.

Let us prove the existence of the limit (2.10). Since $\left\|\exp (i H t) J U_{0}(t)\right\|=1$, it suffices to verify the convergence in $(2.10)$ on the dense set $f \in \mathscr{D} \equiv \mathscr{D}_{m_{2}}$. Let $\zeta\left(x_{2}\right)$ be some smooth function, which is equal to $x_{2}\left|x_{2}\right|^{-2}$ if $\left|x_{2}\right|>1, f_{t}=U_{0}(t) f$,

$$
(\tilde{J} f)\left(x_{1}, x_{2}\right)=\psi\left(x_{1}, x_{2}\right)\left[1+2^{-1} a x_{1}^{2}\left\langle\zeta\left(x_{2}\right), \nabla_{2}\right\rangle_{2}\right] f\left(x_{2}\right) \text {. }
$$

It is convenient for us - and this is the main idea in the proof of the existence of the WO $w$ - to consider initially the expression

$$
\lim _{t \rightarrow \infty} \exp (i H t) \tilde{J} U_{0}(t) f, \quad f \in \mathscr{D} .
$$

First let us show that the existence of the limit (3.4) implies the existence of the WO $w$. Note that

$$
\left\|(J-\tilde{J}) U_{0}(t) f\right\|^{2}=\int d x_{2} u^{2}\left(x_{2}\right)\left|\left\langle\zeta\left(x_{2}\right), \nabla_{2} f_{t}\left(x_{2}\right)\right\rangle_{2}\right|^{2}
$$

and $u\left(x_{2}\right)=2^{-1} a\|\tilde{\psi}\|_{1}=O\left(\left|x_{2}\right|^{2 a}\right)$ by the first condition (3.2). Since $D_{2} U_{0}(t)$ $=U_{0}(t) D_{2}, D_{2} f \in \mathscr{D}$ and $u\left(x_{2}\right) \zeta\left(x_{2}\right)=O\left(\left|x_{2}\right|^{-1+2 a}\right)$ the convergence of the expression (3.5) to zero when $t \rightarrow \infty$ follows from the condition $2 a<1$ and the equality (2.8).

For the proof of the existence of the limit (3.4) it is obviously sufficient to show that

$$
\begin{aligned}
\| \frac{d}{d t} & {\left[\exp (i H t) \tilde{J} U_{0}(t) f\right] \| } \\
& =\left\|\left[H \tilde{J}+\tilde{J} \Delta_{2}-\tilde{J} \mathbf{l}\left(-i \nabla_{2}, t\right)\right] f_{t}\right\|=O\left(t^{-1-\varepsilon}\right) .
\end{aligned}
$$

We shall evaluate $\left(H \tilde{J} f_{t}\right)\left(x_{1}, x_{2}\right)$ taking sufficiently large $\left|x_{2}\right|$. Since the function $f_{t}$ does not depend on the variable $x_{1}$ and $-\Delta_{1} \psi+q \psi=\lambda \psi, \lambda=\lambda\left(x_{2}\right)$, then by definition (3.3)

$$
\left(-\Delta_{1}+q\right) \tilde{J} f_{t}=\lambda \tilde{J} f_{t}-a\left[2\left\langle\nabla_{1} \psi, x_{1}\right\rangle_{1}+m_{1} \psi\right]\left\langle\zeta, \nabla_{2} f_{t}\right\rangle_{2} .
$$

Taking second derivatives of the products $\psi f_{t}$ and $\tilde{\psi}\left\langle\zeta, \nabla_{2} f_{t}\right\rangle_{2}$ in the variable $x_{2}$, we find that

$$
\begin{aligned}
\Delta_{2} \tilde{J} f_{t}= & \left(\Delta_{2} \psi\right) f_{t}+2^{-1} a\left(\Delta_{2} \tilde{\psi}\right)\left\langle\zeta, \nabla_{2} f_{t}\right\rangle_{2} \\
& +2\left\langle\nabla_{2} \psi, \nabla_{2} f_{t}\right\rangle_{2}+a\left\langle\nabla_{2} \tilde{\psi}, \nabla_{2}\left\langle\zeta, \nabla_{2} f_{t}\right\rangle_{2}\right\rangle_{2}+\psi \Delta_{2} f_{t} \\
& +2^{-1} a \tilde{\psi} \Delta_{2}\left\langle\zeta, \nabla_{2} f_{t}\right\rangle_{2} .
\end{aligned}
$$


Let us check that the norms of the first, second and fourth summands in the right hand side of (3.8) decrease as $t^{-1-\varepsilon}$ when $t \rightarrow \infty$, i.e.

$$
\begin{aligned}
& \left\|\left(\Delta_{2} \psi\right) f_{t}\right\|+\left\|\left(\Delta_{2} \tilde{\psi}\right)\left\langle\zeta, \nabla_{2} f_{t}\right\rangle_{2}\right\| \\
& \quad+\left\|\left\langle\nabla_{2} \tilde{\psi}, \nabla_{2}\left\langle\zeta, \nabla_{2} f_{t}\right\rangle_{2}\right\rangle_{2}\right\|=O\left(t^{-1-\varepsilon}\right) .
\end{aligned}
$$

We investigate first $\left(\Delta_{2} \psi\right) f_{t}$. Since the function $f_{t}$ depends only on the variable $x_{2}$, then due to the fourth condition (3.2) $\left\|\left(\Delta_{2}\right) f_{t}\right\|=\left\|v f_{t}\right\|_{2}$, where $v\left(x_{2}\right)=\left\|\Delta_{2} \psi\right\|_{1}$ $=O\left(\left|x_{2}\right|^{-1-\varepsilon}\right)$. The relation (2.7) ensures now that $\left\|v f_{t}\right\|_{2}=O\left(t^{-1-\varepsilon}\right)$. The second and third summands in the left hand side of (3.9) may be considered quite in the same way if we apply correspondingly the third and second conditions (3.2) and additionally take into account that $2 a<1$, that the operators $\nabla_{2}$ and $U_{0}(t)$ commute and that the set $\mathscr{D}$ is invariant with respect to action of components of the operator $\nabla_{2}$.

Let us show that the last summand in the right hand side of (3.8) behaves like $2^{-1} a \tilde{\psi}\left\langle\zeta, \nabla_{2} \Delta_{2} f_{t}\right\rangle_{2}$, when $t \rightarrow \infty$, i.e.

$$
\left\|\tilde{\psi}\left[\Delta_{2}\left\langle\zeta, \nabla_{2} f_{t}\right\rangle_{2}-\left\langle\zeta, \nabla_{2} \Delta_{2} f_{t}\right\rangle_{2}\right]\right\|=O\left(t^{-1-\varepsilon}\right) .
$$

One can rewrite the left-hand side of (3.10) in the form

$$
\left\|\tilde{v}\left[\Delta_{2}\left\langle\zeta, \nabla_{2} f_{t}\right\rangle_{2}-\left\langle\zeta, \nabla_{2} \Delta_{2} f_{t}\right\rangle_{2}\right]\right\|_{2},
$$

where $\tilde{v}\left(x_{2}\right)=\|\tilde{\psi}\|_{1}=O\left(\left|x_{2}\right|^{2 a}\right)$ due to the first condition (3.2). Therefore, if in the product $\left\langle\zeta, \nabla_{2} f_{t}\right\rangle_{2}$ we differentiate the factor $\zeta$ at least once with respect to $x_{2}$, there arise for the functions $D_{2} f_{t}$ (or $D_{2}^{2} f_{t}$ ) coefficients decreasing as $\left|x_{2}\right|^{-3+2 a}$ (or $\left|x_{2}\right|^{-2+2 a}$ ) when $\left|x_{2}\right| \rightarrow \infty$. Since $2 a<1$ by virtue of the equality (2.7), this implies that the norm of the corresponding summand decreases as $t^{-1-\varepsilon}$ when $t \rightarrow \infty$. This proves the equality (3.10). Relations (3.8)-(3.10) ensure that

$$
\left\|\Delta_{2} \tilde{J} f_{t}-2\left\langle\nabla_{2} \psi, \nabla_{2} f_{t}\right\rangle_{2}-\tilde{J} \Delta_{2} f_{t}\right\|=O\left(t^{-1-\varepsilon}\right) .
$$

The equalities (3.7) and (3.11) show that

$$
\begin{aligned}
& \|\left[H \tilde{J}+\tilde{J} \Delta_{2}-\tilde{J} \mathbf{l}\left(-i \nabla_{2}, t\right) f_{t} \|\right. \\
& \leqq \\
& \quad+\left\langle\left[a\left(2\left\langle\nabla_{1} \psi, x_{1}\right\rangle_{1}+m_{1} \psi\right) \zeta+2 \nabla_{2} \psi\right], \nabla_{2} f_{t}\right\rangle_{2} \| \\
& \quad+\left\|\psi\left[\lambda\left(x_{2}\right)-\mathbf{l}\left(-i \nabla_{2}, t\right)\right] f_{t}\right\| \\
& \quad+2^{-1} a\left\|\tilde{\psi}\left\langle\zeta,\left[\lambda\left(x_{2}\right)-\mathbf{l}\left(-i \nabla_{2}, t\right)\right] \nabla_{2} f_{t}\right\rangle_{2}\right\|+O\left(t^{-1-\varepsilon}\right) .
\end{aligned}
$$

The last condition of (3.2) and equality (2.7) imply that the first summand in the right hand side of (3.12) decreases as $t^{-1-\varepsilon}$ if $t \rightarrow \infty$. The second summand in (3.12) does not exceed $t^{-1-\varepsilon}$ due to the fact that by construction of the function 1 the relation (2.6), (where the role of $q$ is played by $\lambda$ ) is valid. The same considerations together with the first condition of (3.2) show that the third summand in (3.2) is also $O\left(t^{-1-\varepsilon}\right)$. Thus the relation (3.6) and consequently the existence of the WO $w$ are proved. We shall summarize the previous results in the following

Theorem 2. Let the functions $\lambda\left(x_{2}\right)$ and $\psi\left(x_{1}, x_{2}\right)$ satisfy respectively conditions (2.4) (where $\alpha>0$ ) and (3.2) (where $a \in[0,1 / 2)$ ). Then the WO $w$ exists. 


\section{Orthogonality of Channels}

Assume that the WO $W$ and $w$, defined by relations (2.1) and (2.10), exist. We shall show that the subspaces $R(W)$ and $R(w)$ are orthogonal (orthogonality of channels of scattering). We do not assume now the function $\psi\left(x_{1}, x_{2}\right)$, by which the identification $J$ is constructed, to be an eigenfunction of the operator $h\left(x_{2}\right), U_{0}(t)$ is an arbitrary family of unitary operators in the space $\mathscr{H}_{2}$. We do not need also either conditions (2.4) or (3.2). Some assumptions on $\psi\left(x_{1}, x_{2}\right)$, which are necessary for the proof of the orthogonality of $R(W)$ and $R(w)$, will be made later. These assumptions correspond also to the behavior (3.1) of the function $\psi\left(x_{1}, x_{2}\right)$ when $\left|x_{2}\right| \rightarrow \infty$. Thus, conditions for the existence of the WO $w$ and conditions for the orthogonality of channels are essentially different.

For the proof of orthogonality of channels it suffices to check that

$$
\left(\exp \left(-i H_{0} t\right) f, J U_{0}(t) g\right) \rightarrow 0, \quad t \rightarrow \infty,
$$

where the function $f$ belongs to some dense subset of $\mathscr{H}$ and $g \in \mathscr{H}_{2}$. Let $f(x)$ $=f_{1}\left(x_{1}\right) f_{2}\left(x_{2}\right)$, where $f_{1} \in \mathscr{E}, \overline{\mathscr{E}}=\mathscr{H}_{1}, f_{2} \in \mathscr{H}_{2}$. The linear combinations of functions $f$ of this type are dense in $\mathscr{H}$ and

$$
\begin{gathered}
\left(\exp \left(-i H_{0} t\right) f, J U_{0}(t) g\right)=\int d x_{2}\left(\exp \left(i \Delta_{2} t\right) f_{2}\right)\left(x_{2}\right) \\
. \overline{\left(U_{0}(t) g\right)\left(x_{2}\right)} \int\left(\exp \left(i \Delta_{1} t\right) f_{1}\right)\left(x_{1}\right) \psi\left(x_{1}, x_{2}\right) d x_{1} .
\end{gathered}
$$

Using Schwarz inequality and unitarity of the operators $\exp \left(i \Delta_{2} t\right)$ and $U_{0}(t)$ in $\mathscr{H}_{2}$, one can estimate this expression by

$$
\|f\|_{2}\|g\|_{2} \sup _{x_{2}}\left|\int\left(\exp \left(i \Delta_{1} t\right) f_{1}\right)\left(x_{1}\right) \psi\left(x_{1}, x_{2}\right) d x_{1}\right| .
$$

Let us now make precise the choice of the set $\mathscr{E}: \mathscr{E}$ consists of linear combinations of functions $f_{1}\left(x_{1}\right)=\exp \left[-2^{-1}\left(x_{1}-\sigma\right)^{2}\right]$ for all $\sigma \in \mathbb{R}^{m_{1}}$. It is well known (see, for example, [10]) that $\overline{\mathscr{E}}=\mathscr{H}_{1}$ and

$$
\left(\exp \left(i \Delta_{1} t\right) f_{1}\right)\left(x_{1}\right)=(1+2 i t)^{-m_{1} / 2} \exp \left[b(t)\left(x_{1}-\sigma\right)^{2}\right],
$$

where $b(t)=-2^{-1}(1+2 i t)^{-1}$. Thus for the proof of the relation (4.1) it suffices to check that for any fixed $\sigma \in \mathbb{R}^{m_{1}}$

$$
A\left(x_{2}, t\right) \equiv(1+2 i t)^{-m_{1} / 2} \int \exp \left[b(t)\left(x_{1}-\sigma\right)^{2}\right] \psi\left(x_{1}, x_{2}\right) d x_{1}
$$

goes to zero uniformly in $x_{2}$ when $t \rightarrow \infty$, i.e.

$$
\lim _{t \rightarrow \infty} \sup _{x_{2}}\left|A\left(x_{2}, t\right)\right|=0 \text {. }
$$

For the proof of the equality (4.3) we must study the cases $m_{1}=1, m_{1}=2$ and $m_{1} \geqq 3$ separately. If $m_{1}=1$ assume that

$$
\begin{aligned}
& \lim _{\left|x_{2}\right| \rightarrow \infty} \int\left|\psi_{x_{1}}^{\prime}\left(x_{1}, x_{2}\right)\right| d x_{1}=0, \\
& \sup _{\left|x_{2}\right| \leqq N} \int\left|\psi\left(x_{1}, x_{2}\right)\right| d x_{1} \leqq C(N),
\end{aligned}
$$

where $N$ is an arbitrary positive number. The inequalities (4.5) and $\left|\exp \left[b\left(x_{1}-\sigma\right)^{2}\right]\right|<1$ ensure that $|A| \leqq C(N) t^{-1 / 2}$ if $\left|x_{2}\right| \leqq N$. On the other hand, 
integrating by parts one finds that

$$
\begin{aligned}
\int \exp & {\left[b\left(x_{1}-\sigma\right)^{2}\right] \psi\left(x_{1}, x_{2}\right) d x_{1} } \\
& =-\int\left(\int_{0}^{x_{1}-\sigma} \exp \left(b y^{2}\right) d y\right) \psi_{x_{1}}^{\prime}\left(x_{1}, x_{2}\right) d x_{1} .
\end{aligned}
$$

Since

$$
\left|\int_{0}^{x} \exp \left(b y^{2}\right) d y\right| \leqq C t^{1 / 2}
$$

by (4.2), (4.4) the function $A\left(x_{2}, t\right)$ tends to zero uniformly in $t$ when $\left|x_{2}\right| \rightarrow \infty$. This implies the relation (4.3) (if $m_{1}=1$ ).

Now it is convenient for us to investigate the case $m_{1} \geqq 3$. Let $n$ be equal to the smallest integer that is equal to or larger than $4^{-1} m_{1}$; it is clear that $4^{-1} m_{1} \leqq n$ $<2^{-1} m_{1}$. Let the function $\psi\left(x_{1}, x_{2}\right)$ have all partial derivatives up to the order $n$ with respect to the variables $x_{1}$ and for $l \equiv|x| \leqq n$

$$
\begin{array}{r}
\sup _{x}\left|D_{1}^{\alpha} \psi\left(x_{1}, x_{2}\right)\right| \leqq C, \\
\sup _{x_{2}} \int\left|x_{1}\right|^{-2 n+l}\left|D_{1}^{\alpha} \psi\left(x_{1}, x_{2}\right)\right| d x_{1} \leqq C .
\end{array}
$$

Take in $\mathbb{R}^{m_{1}}$ spherical coordinates with center in the point $\sigma$, i.e. $x_{1}=(\varrho, \omega)$, $\left|x_{1}-\sigma\right|=\varrho, d x_{1}=\varrho^{m_{1}-1} d \varrho d \omega$, and let

$$
\hat{\psi}\left(\varrho, x_{2}\right)=\int d \omega \psi\left((\varrho, \omega), x_{2}\right) .
$$

Applying the formula $d\left(\exp \left(b \varrho^{2}\right)\right)=2 b \varrho \exp \left(b \varrho^{2}\right) d \varrho$, we integrate in the integral

$$
\int \exp \left(b\left(x_{1}-\sigma\right)^{2}\right) \psi\left(x_{1}, x_{2}\right) d x_{1}=\int_{0}^{\infty} \exp \left(b \varrho^{2}\right) \varrho^{m_{1}-1} \hat{\psi}\left(\varrho, x_{2}\right) d \varrho
$$

$n$ times by parts. The first integration by parts gives the equality

$$
\begin{aligned}
& \int_{0}^{\infty} \exp \left(b \varrho^{2}\right) \varrho^{m_{1}-1} \hat{\psi}\left(\varrho, x_{2}\right) d \varrho \\
&=\left.2^{-1} b^{-1} \varrho^{m_{1}-2} \hat{\psi}\left(\varrho, x_{2}\right) \exp \left(b \varrho^{2}\right)\right|_{0} ^{\infty}-2^{-1} b^{-1} \int_{0}^{\infty} \exp \left(b \varrho^{2}\right) \\
& \cdot\left[\left(m_{1}-2\right) \varrho^{m_{1}-3} \hat{\psi}\left(\varrho, x_{2}\right)+\varrho^{m_{1}-2} \hat{\psi}_{\varrho}^{\prime}\left(\varrho, x_{2}\right)\right] d \varrho .
\end{aligned}
$$

After the $n^{\text {th }}$ integration by parts we get that for some numbers $\beta_{l}$

$$
\begin{aligned}
& \int_{0}^{\infty} \exp \left(b \varrho^{2}\right) \varrho^{m_{1}-1} \hat{\psi}\left(\varrho, x_{2}\right) d \varrho \\
& \quad=b^{-n} \sum_{l=0}^{n} \beta_{l} \int_{0}^{\infty} \exp \left(b \varrho^{2}\right) \varrho^{m_{1}-2 n+l-1} \hat{\psi}_{\varrho}^{(l)}\left(\varrho, x_{2}\right) d \varrho .
\end{aligned}
$$

The non-integral terms, corresponding to $\varrho=\infty$, are equal to zero by the condition (4.6) and the inequality $\operatorname{Re} b<0$. If $\varrho=0$ the non-integral terms turn out to be zero, since they contain the factor $\varrho^{s}$, where $s \geqq m_{1}-2 n>0$. Relations (4.2), (4.8), (4.10) 
and the obvious inequality $|b| \leqq C t$ imply that

$$
\left|A_{2}\right| \leqq C t^{-m_{1} / 2+n} \sum_{l=0}^{n} \int_{0}^{\infty} \varrho^{m_{1}-2 n+l-1}\left|\hat{\psi}_{\varrho}^{(l)}\left(\varrho, x_{2}\right)\right| d \varrho .
$$

Since $2 n<m_{1}$, the proof of (4.3) is reduced to checking the uniform boundedness in $x_{2}$ of each integral in (4.11). Returning to cartesian coordinates we find that

$$
\int_{0}^{\infty} \varrho^{m_{1}-2 n+l-1}\left|\hat{\psi}_{\varrho}^{(l)}\left(\varrho, x_{2}\right)\right| d \varrho \leqq \sum_{|x|=l} \int\left|x_{1}-\sigma\right|^{-2 n+l}\left|D_{1}^{\chi} \psi\left(x_{1}, x_{2}\right)\right| d x_{1} .
$$

Split each integral in the right hand side of (4.12) into two parts: over regions $\left|x_{1}\right|<2 \sigma$ and $\left|x_{1}\right|>2 \sigma$. By condition (4.6) and inequality $2 n<m_{1}$, the first of these integrals is bounded by a constant. The integral over the region $\left|x_{1}\right|>2 \sigma$ clearly does not exceed the integral in (4.7), which is by assumption bounded uniformly in $x_{2}$. Therefore the relation (4.3) is proved also for $m_{1} \geqq 3$.

Let now $m_{1}=2$; set $n=1$. We repeat the considerations carried out earlier for $m_{1} \geqq 3$. Integrating by parts we come back again to the equality (4.9). But now the non-integral term does not vanish at $\varrho=0$, so that the equality (4.9) leads to the estimate

$$
|A| \leqq C\left[\left|\psi\left(\sigma, x_{2}\right)\right|+\int\left|x_{1}-\sigma\right|^{-1}\left|\nabla_{1} \psi\left(x_{1}, x_{2}\right)\right| d x_{2}\right] .
$$

Assume that for $m_{1}=2$

$$
\left.\begin{array}{rl}
\lim _{\left|x_{2}\right| \rightarrow \infty} D_{1}^{\alpha} \psi\left(x_{1}, x_{2}\right) & =0, \quad|x|=0,1, \\
\lim _{\left|x_{2}\right| \rightarrow \infty} \int\left|x_{1}\right|^{-1}\left|\nabla_{1} \psi\left(x_{1}, x_{2}\right)\right| d x_{1} & =0 .
\end{array}\right\}
$$

Estimating the integral in (4.13) as in the case $m_{1} \geqq 3$ and using conditions (4.14) we find that the expression (4.13) goes to zero if $\left|x_{2}\right| \rightarrow \infty$ uniformly in $t$. Let also for $m_{1}=2$ the condition (4.5) be fulfilled. Then as in the case $m_{1}=1$ one finds that $|A| \leqq C(N) t^{-1},\left|x_{2}\right| \leqq N$. This ensures that the relation (4.3) is valid also when $m_{1}=2$. So we have proved

Theorem 3. Let the WO $W$ and $w$ be defined by the relations (2.1) and (2.10), where $U_{0}(t)$ is an arbitrary family of unitary operators in the space $\mathscr{H}_{2}$ and the isometric identification $J$ is constructed by formula (2.9). Assume the existence of limits in (2.1) and (2.10). With respect to the function $\psi\left(x_{1}, x_{2}\right)$ assume that for $m_{1}=1$ conditions (4.4), (4.5) are fulfilled, for $m_{1}=2$ conditions (4.5), (4.14), and for $m_{1} \geqq 3$ conditions (4.6), (4.7). Then the subspaces $R(W)$ and $R(w)$ are orthogonal.

Let for sufficiently large $\left|x_{2}\right|$ the operator $h\left(x_{2}\right)$ have several eigenvalues (or the single eigenvalue be degenerate). We denote by $w^{1}$ and $w^{2}$ the WO, constructed by formula (2.10) with respect to some orthogonal eigenfunctions $\psi^{1}$ and $\psi^{2}$ of the operator $h\left(x_{2}\right)$. Then the subspaces $R\left(w^{1}\right)$ and $R\left(w^{2}\right)$ are orthogonal. Actually, the unitarity of operators $\exp (i H t)$ implies that

$$
\left(w^{1} f, w^{2} g\right)=\lim _{t \rightarrow \infty}\left(J^{1} U_{0}^{1}(t) f, J^{2} U_{0}^{2}(t) g\right)=\lim _{t \rightarrow \infty}\left(V U_{0}^{1}(t) f, U_{0}^{2}(t) g\right)_{2},
$$

where $V$ is multiplication by the function $v\left(x_{2}\right)=\left(\psi_{1}, \psi_{2}\right)_{1}$. Since $v\left(x_{2}\right)=0$ for sufficiently large $\left|x_{2}\right|$ the right-hand side of (4.15) goes to zero by virtue of (2.8). 


\section{The Discrete Spectrum}

As was pointed out in $\S 1$, for potentials considered in the present paper the negative discrete spectrum of the operator $H$ is infinite. The condition for infinitude of the discrete spectrum of Schrödinger operators, obtained in this framework, cannot be deduced directly from the known criteria (see, for example, $[11,12])$, in which, roughly speaking, the inequality $q(x)<c|x|^{-2+\varepsilon}, c<0, \varepsilon>0$, is assumed. In the study of the discrete spectrum we shall use essentially simpler assumptions than for constructing scattering theory in $\S 3,4$.

Theorem 4. Let, at least for sufficiently large $\left|x_{2}\right|$, the operator $h\left(x_{2}\right)=-\Delta_{1}$ $+q\left(x_{1}, x_{2}\right)$ in the space $\mathscr{H}_{1}$ have a negative eigenvalue $\lambda\left(x_{2}\right)$, where $\lambda\left(x_{2}\right)<c\left|x_{2}\right|^{-2+\varepsilon}$, $c<0, \varepsilon>0$, and let the corresponding real-valued normalized eigenfunction $\psi\left(x_{1}, x_{2}\right)$ satisfy $d\left(x_{2}\right) \equiv\left\|\nabla_{2} \psi\right\|_{1}=O\left(\left|x_{2}\right|^{-1}\right)$. Then the multiplicity of the negative spectrum of the operator $H$ is infinite.

Proof. It suffices to construct an infinite-dimensional linear manifold $\mathscr{M}, \mathscr{M} \subset \mathscr{H}$, such that $(H u, u)<0$ for $u \in \mathscr{M}$. Let us evaluate

$$
(H u, u)=\int\left(\left|\nabla_{1} u\right|^{2}+\left|\nabla_{2} u\right|^{2}+q u^{2}\right) d x
$$

for the functions $u\left(x_{1}, x_{2}\right)=\psi\left(x_{1}, x_{2}\right) f\left(x_{2}\right)$, where $f=\bar{f}$. Since $\nabla_{1} u=\left(\nabla_{1} \psi\right) f$ and $\int\left(\left|\nabla_{1} \psi\right|^{2}+q \psi^{2}\right) d x_{1}=\lambda\left(x_{2}\right)$, then

$$
\int\left(\left|\nabla_{1} u\right|^{2}+q u^{2}\right) d x=\int \lambda\left(x_{2}\right) f^{2}\left(x_{2}\right) d x_{2} .
$$

Differentiation of the condition of normalization $\int \psi^{2}\left(x_{1}, x_{2}\right) d x_{1}=1$ provides that $\int \psi \nabla_{2} \psi d x_{1}=0$. This implies that

$$
\begin{aligned}
\int\left|\nabla_{2} u\right|^{2} d x & =\int\left(\psi^{2}\left|\nabla_{2} f\right|^{2}+2 f \psi\left\langle\nabla_{2} f, \nabla_{2} \psi\right\rangle_{2}+f^{2}\left|\nabla_{2} \psi\right|^{2}\right) d x \\
& =\int\left(\left|\nabla_{2} f\right|^{2}+d^{2} f^{2}\right) d x_{2} .
\end{aligned}
$$

The equalities (5.1)-(5.3) show that, for $u\left(x_{1}, x_{2}\right)=\psi\left(x_{1}, x_{2}\right) f\left(x_{2}\right)$ with $\mu\left(x_{2}\right)=\lambda\left(x_{2}\right)+d^{2}\left(x_{2}\right)$, the relation

$$
(H u, u)=\int\left(\left|\nabla_{2} f\right|^{2}+\mu f^{2}\right) d x_{2}
$$

is valid.

Consider now the operator $\hat{h}=-\Delta_{2}+\mu\left(x_{2}\right)$ in the space $\mathscr{H}_{2}$. By the condition $\mu\left(x_{2}\right)<c\left|x_{2}\right|^{-2+\varepsilon}, c<0, \varepsilon>0$, the multiplicity of negative spectrum of the operator $\hat{h}$ is infinite. Consequently, there exists a linear manifold $\hat{\mathscr{M}}, \hat{\mathscr{M}} \subset \mathscr{H}_{2}$ such that $(\hat{h} f, f)<0$ and $f=\bar{f}$ for $f \in \hat{M}$. By $(5.4)(H u, u)=(\hat{h} f, f)$ so that one can choose $\mathscr{M}$ to be the set of functions of the form $u\left(x_{1}, x_{2}\right)=\psi\left(x_{1}, x_{2}\right) f\left(x_{2}\right)$, where $f \in \hat{\mathscr{M}}$.

Corollary. If, under the assumptions of Theorem $4 q(x) \rightarrow 0$ when $|x| \rightarrow \infty$, then the operator $H$ has an infinite number of negative eigenvalues, accumulating at the point zero.

\section{Classes of Potentials}

Let us describe here explicitly two classes of potentials $q(x)$, for which all previous assumptions on the behavior of eigenfunctions $\psi\left(x_{1}, x_{2}\right)$ and eigenvalues $\lambda\left(x_{2}\right)$ of 
the operator $h\left(x_{2}\right)=-\Delta_{1}+q\left(x_{1}, x_{2}\right)$ are valid. These classes are analogous to the corresponding sets of functions, depending on time $t$ introduced in [8].

Suppose that for sufficiently large $\left|x_{2}\right|$

$$
q\left(x_{1}, x_{2}\right)=\left|x_{2}\right|^{-2 a} p\left(\left|x_{2}\right|^{-a} x_{1}\right), \quad a \geqq 0 .
$$

Then

$$
h\left(x_{2}\right)=\left|x_{2}\right|^{-2 a} T_{a}\left(x_{2}\right) h(1) T_{a}^{*}\left(x_{2}\right),
$$

where $T_{a}\left(x_{2}\right)$ is the unitary operator of dilations in the space

$$
\mathscr{H}_{1}:\left(T_{a}\left(x_{2}\right) g\right)\left(x_{1}\right)=\left|x_{2}\right|^{\frac{-m_{1} a}{2}} g\left(\left|x_{2}\right|^{-a} x_{1}\right) \text {. }
$$

Let the operator $h(1)$ have an eigenvalue $\lambda$ with an eigenfunction $\varphi\left(x_{1}\right)$. The relation (6.2) implies that

$$
\psi\left(x_{1}, x_{2}\right)=\left|x_{2}\right|^{\frac{-m_{1} a}{2}} \varphi\left(\left|x_{2}\right|^{-a} x_{1}\right)
$$

is an eigenfunction of the operator $h\left(x_{2}\right)$ with eigenvalue $\lambda\left(x_{2}\right)=\left|x_{2}\right|^{-2 a} \lambda$. Thus, for potentials of the form (6.1) the condition (2.4) (by $\alpha=2 a$ ) holds, and the relation (3.1) turns out to be an equality for sufficiently large $\left|x_{2}\right|$. Under the assumption $\left\|(|\varphi|+|\nabla \varphi|+|\Delta \varphi|)\left(1+x_{1}^{2}\right)\right\|_{1}<\infty$ the first four inequalities (3.2) can be verified by explicit calculation. Moreover, for functions $\psi\left(x_{1}, x_{2}\right)=\left|x_{2}\right|^{\frac{-m_{1} a}{2}} \varphi\left(\left|x_{2}\right|^{-a} x_{1}\right)$ the expression in the last condition (3.2) is identically equal to zero. Therefore, if $a \in(0,1 / 2)$ for potentials $(6.1)$ the WO $w$ exists by Theorem 2. All conditions of $\S 4$ depend only on the existence and certain decrease at infinity of some number of derivatives of the function $\varphi$.

By virtue of Theorem 1 for potentials (6.1) the WO $W$ exists if $\left|p\left(x_{1}\right)\right|$ $\leqq C\left(1+\left|x_{1}\right|\right)^{-\alpha_{1}}$, where $\alpha_{1}>(1-2 a)(1-a)^{-1}, \alpha_{1} \geqq 0$. By Theorem 3 the ranges of the operators $W$ and $w$ are orthogonal so that the WO $W$ is not complete for such potentials. Since the function $p\left(x_{1}\right)$ may decrease slower than $\left|x_{2}\right|^{-2}$, the operator $h(1)$ can have infinite discrete spectrum. Therefore for such potentials there exist in general an infinite number of WO $w^{i}$ with orthogonal ranges. By virtue of Theorem 4 the multiplicity of the negative spectrum of the operator $H$ is infinite if $a<1$ and the operator $h(1)$ has a negative eigenvalue $\lambda$.

In particular, for homogeneous functions $p\left(x_{1}\right)=\omega\left(x_{1}\left|x_{1}\right|^{-1}\right)\left|x_{1}\right|^{-\alpha_{1}}$ the relation (6.1) takes the form ${ }^{1}$

$$
q\left(x_{1}, x_{2}\right)=\omega\left(x_{1}\left|x_{1}\right|^{-1}\right)\left|x_{1}\right|^{-\alpha_{1}}\left|x_{2}\right|^{-\alpha_{2}},
$$

where $\alpha_{2}=a\left(2-\alpha_{1}\right)$ and the condition $2 a<1$ transforms into the inequality $2^{-1} \alpha_{1}+\alpha_{2}<1$. Therefore when $\alpha_{1}+\alpha_{2}>1, \alpha_{j} \geqq 0,2^{-1} \alpha_{1}+\alpha_{2}<1$ the WO $W$ exists but it is not complete if the operator $h(1)$ has an eigenvalue. This implies that for potentials of the form (6.3) the conditions (1.3) of completeness of WO cannot be improved.

1 If $\alpha_{1}=\alpha_{1}\left(m_{1}\right)$ is not too large the singularity of $q\left(x_{1}, x_{2}\right)$ at $x_{1}=0$ does not violate the selfadjointness of operators $h(1)$ and $H$ 
The second class of potentials, to which the results of the present paper are applicable, consists of functions

$$
q(x)=p\left(x_{1}\right) r\left(x_{2}\right),
$$

where $m_{1}=1, r\left(x_{2}\right) \sim c\left|x_{2}\right|^{-\alpha_{2}},\left|x_{2}\right| \rightarrow \infty, \alpha_{2} \in(0,1 / 2)$ and the function $p\left(x_{1}\right)$ goes to zero sufficiently quickly when $\left|x_{1}\right| \rightarrow \infty$. Under the assumption $\left|p\left(x_{1}\right)\right| \leqq C\left(1+\left|x_{1}\right|\right)^{-\alpha_{1}}, \alpha_{1}>1$, the WO $W$ exists. If, moreover, $\alpha_{1}>2$, then obviously $\alpha_{1}+2^{-1} \alpha_{2}>1,2^{-1} \alpha_{1}+\alpha_{2}>1$, but the conditions of completeness (1.3) are violated still because for $m_{1}=1$ they require also the inequality $2 \alpha_{2}>1$. In particular, for $m_{1}=m_{2}=1$ and a function $p$ with compact support the function $q$ is different from zero only in some strip, parallel to the axis $x_{1}=0$. Therefore the scattering by the potential, contained in the strip, is qualitatively dependent on the speed of falloff of the potential along the strip (the critical value is $\left|x_{2}\right|^{-1 / 2}$ ).

For verification of the assumptions of $\S 3-5$ we need some information about the behavior of eigenvalues and eigenfunctions of the one-dimensional operator $h_{2}=-\frac{d^{2}}{d x^{2}}+r p(x)$ if $P \equiv \int p(x) d x<0$ and $r \rightarrow 0, r>0$. For sufficiently small $r$ the operator $h(r)$ has a unique (negative) eigenvalue $\lambda(r)=-s^{2}(r)$; thus, by virtue of the results of the paper [13]

$$
s(r)=-2^{-1} P r+O\left(r^{2}\right) .
$$

Moreover, for sufficiently rapid falloff of $p(x)$ one can differentiate the asymptotics (6.5) a sufficient number of times. Denote by $\psi(x, r)$ the real-valued normalized eigenfunction of the operator $h_{r} ; f(x, r), g(x, r)$ are the solutions of the equation $h_{r} \psi=\lambda(r) \psi \quad$ with asymptotics $f(x, r) \sim \exp [-s(r) x]$ when $x \rightarrow+\infty$ and $g(x, r) \sim \exp [s(r) x]$ when $x \rightarrow-\infty$. In the paper [14] the estimate

$$
\left|f(x, r)-\mathrm{e}^{-s(r) x}\right| \leqq C \mathrm{e}^{-s(r) x} r \int_{x}^{\infty}(1+|y|)|p(y)| d y, \quad x \geqq 0,
$$

and a similar inequality for $g(x, r)$ are proved. Moreover, using wellknown [14] integral representations for the functions $f$ and $g$ and the asymptotics (6.5) one can show that the relations $f(x, r) \sim \exp [-s(r) x], g(x, r) \sim \exp [s(r) x], r \rightarrow 0$ can be differentiated with respect to the variables $x$ and $r$. We do not dwell on the precise meaning of this assertion. It suffices for us to differentiate twice with respect to $r$ and once with respect to $x$. Since $\lambda(r)=-s^{2}(r)$ is an eigenvalue of the operator $h_{r}$, the functions $\psi, f$ and $g$ are proportional. This implies that

$$
\psi(x, r) \sim s^{1 / 2}(r) \exp [-s(r)|x|], \quad r \rightarrow 0 .
$$

Let us go back to the study of the operator $H$ with the potential (6.4). Assume that for sufficiently large $\left|x_{2}\right|$

$$
r\left(x_{2}\right)=c\left|x_{2}\right|^{-\alpha_{2}}+\tilde{r}\left(x_{2}\right), \quad c>0, \quad \alpha_{2} \in(0,1 / 2),
$$

the function $r\left(x_{2}\right)$ has $k=\left[\left(2 \alpha_{2}\right)^{-1}\right]+2$ continuous derivatives and

$$
D_{2}^{\varkappa} \tilde{r}\left(x_{2}\right)=O\left(\left|x_{2}\right|^{-\alpha_{2}-k-\varepsilon}\right), \quad|x| \leqq k, \quad \varepsilon>0 .
$$

Not giving precise assumptions on the function $p\left(x_{1}\right)$ we mention only that we require more rapid falloff of the function $p\left(x_{1}\right)$ for smaller $\alpha_{2}$, and $P<0$. 
Asymptotics (6.5), (6.6) and assumptions (6.7), (6.8) ensure that the function $\lambda\left(x_{2}\right)$ satisfies the condition (2.4), and that the relation (3.1), where $a=\alpha_{2}$, $\varphi\left(x_{1}\right)=2^{-1 / 2}(-c P)^{1 / 2} \exp \left(2^{-1} c P\left|x_{1}\right|\right)$, is valid for the function $\psi\left(x_{1}, x_{2}\right)$. The relation (3.1) implies the validity of the conditions (3.2) and (4.4). Therefore by Theorems 2 and 3 for potentials of the form (6.4) there exists the WO $w$ and the ranges of the operators $W$ and $w$ are orthogonal.

If the conditions (6.7), (6.8), where $c>0, \alpha_{2} \in[0,1), k=1, P<0$ and $\int\left(1+\left|x_{1}\right|\right)\left|p\left(x_{1}\right)\right| d x_{1}<\infty$ are fulfilled, then by virtue of Theorem 4 the operator $H$ with the potential (6.4) has infinite discrete spectrum.

In conclusion we note that for potentials (6.4) when $m_{2}=1$ the assertions of Theorems 1-4 are also valid if the bounded function $r\left(x_{2}\right)$ satisfies conditions (6.7), (6.8) only when $x_{2} \rightarrow+\infty$. In this case for constructing the operator $U_{0}(t)$ one may use the operator $-d^{2} / d x^{2}+\lambda\left(x_{2}\right)$ with zero boundary condition in the space $L_{2}(0, \infty)$.

\section{References}

1. Kato, T., Kuroda, S.T.: Rocky Mount. J. Math. 1, 127-171 (1971)

2. Jauch, J.M.: Helv. Phys. Acta 31, 661-684 (1958)

3. Hack, M.N.: Nuovo Cimento 13, 231-236 (1958)

4. Faddeev, L.D.: Tr. MIAN (Russian) 69, 1-121 (1963)

5. Buslaev, V.S., Matveev, V.B.: Teor. Mat. Fis. (Russian) 2, 367-376 (1970)

6. Deich, V.G., Korotyaev, E.L., Yafaev, D.R. : Dokl. AN SSSR (Russian) 235, 749-752 (1977)

7. Deich, V.G., Korotyaev, E.L., Yafaev, D.R.: Notes of Scientific Seminars of the V.A. Steklov Mathematical Institute, Leningrad Branch. Academy of the Sciences of the USSR (in Russian) 73, 35-51 (1977)

8. Yafaev, D.R. : Dokl. AN SSSR (Russian). 243, 1150-1153 (1978)

9. Cook, J.M. : J. Math. Phys. 36, 82-87 (1957)

10. Kato, T.: Perturbation theory for linear operators. Berlin, Heidelberg, New York: Springer 1966

11. Birman, M.Sh.: Mat. Sb. (Russian) 55, 125-174 (1961)

12. Glasman, I.M.: Direct methods of qualitative spectral analysis of singular differential operators (in Russian). Moscow: Fiz. Mat. 1963. (English translation: Jerusalem: Israel Program of Scientific Translations 1966)

13. Simon, B.: Ann. Phys. 97, 279 (1976)

14. Faddeev, L.D.: Tr. MIAN 73, 314-336 (1964)

Communicated by J. Ginibre

Received November 8, 1978 
